\title{
A Study of Temperature Dependence of Thermodynamic Interactions in Polymer Solutions of Infinite Dilution by Equation-of-State Theory
}

\author{
K. K. CHEE \\ Department of Chemistry, University of Malaya, University of Malaya, \\ 59100 Kuala Lumpur, Malaysia \\ (Received April 10, 1990)
}

\begin{abstract}
On the basis of Flory's equation-of-state theory, an expression is developed to characterize the thermodynamic interactions in polymer solutions of infinite dilution. It has been successfully applied to deal with the variations of Flory-Huggins interaction parameter with temperature for polystyrene and polyisobutylene in both good and poor solvents. The shortcomings of the model are discussed.

KEY WORDS Polymer Solutions / Good and Poor Solvents / Polymer-Solvent Interaction Parameter / Exchange Parameters / Equation-ofState Theory / Theta Temperature /
\end{abstract}

The lattice model of polymeric systems originally proposed by Flory and Huggins indeed plays a major role in understanding the thermodynamic properties of polymer solutions as well as the thermodynamic miscibility of polymer and copolymer blends. ${ }^{1,2}$ This classical theory adopts a binary interaction parameter, which, in the present context, is designated by the polymer-solvent interaction parameter, $\chi$, to characterize the exchange interaction energy. Traditionally, $\chi$ is treated as an empirical quantity. As a result, the Flory-Huggins solution thermodynamics fails to elucidate the underlying causes of lower and upper critical solution temperatures (LCST and UCST) and volume change of mixing. However, the recent advances of equation-ofstate theroy which expresses $\chi$ as a function of polymer concentration and temperature, $T$, explicitly, provide the theoretical basis for the foregoing phenomena. ${ }^{3-6}$ In this work, the $\chi-T$ relationships for a number of polymer solutions are examined by the equation-of-state theory. More important, it offers an alternative route to extract the characteristic parameters of this contemporary model as described later.

According to the Flory-Huggins theory, the thermodynamic interactions in polymer solutions are given by

$$
\chi=\mu_{1}^{\mathrm{R}} / R T V_{2}^{2}
$$

where $R$ is the gas constant, $V_{2}$ and $\mu_{1}^{\mathrm{R}}$ are the volume fraction and residual chemical potential respectively. Hereafter, the subscripts 1 and 2 respectively refer to the solvent and polymer in the mixture. By considering the importance of the free volume constribution and the exchange interaction enthalpy and entropy to the free energy of mixing, Flory has derived

$$
\begin{aligned}
\mu_{1}^{\mathrm{R}}= & \frac{M_{1} \bar{v}_{1}}{\tilde{v}_{1}}\left\{P_{1}^{*}\left[3 \tilde{T}_{1} \ln \left(\frac{\tilde{v}_{1}^{1 / 3}-1}{\tilde{v}^{1 / 3}-1}\right)+\left(\frac{1}{\tilde{v}_{1}}-\frac{1}{\tilde{v}}\right)\right]\right. \\
& \left.+\theta_{2}^{2}\left(\frac{X_{12}}{\tilde{v}}-T Q_{12}\right)\right\}
\end{aligned}
$$

where $M_{i}, \bar{v}_{i}, \tilde{v}_{i}, \tilde{T}_{i}$, and $P_{i}^{*}(i=1,2)$ are the molecular weight, specific volume $\left(\mathrm{mlg}^{-1}\right)$, 
reduced volume, reduced temperature, and characteristic pressure of the component $i$ respectively, $\tilde{v}$ is the reduced volume of the solution, and $X_{12}, Q_{12}$ are respectively the characteristic exchange enthalpy and entropy parameters. Here, $\mu_{1}^{\mathbf{R}}$ is related to the concentration of the solution via the contact site fraction of polymer segment

$$
\theta_{2}=\frac{\phi_{2}}{\phi_{2}+\phi_{1} S_{1} / S_{2}}
$$

and the reduced temperature of the solution

$$
\tilde{T}=\frac{\phi_{1} P_{1}^{*} \tilde{T}_{1}+\phi_{2} P_{2}^{*} \tilde{T}_{2}}{\phi_{1} P_{1}^{*}+\phi_{2} P_{2}^{*}-\phi_{1} \theta_{2} X_{12}}
$$

where $\phi_{i}$ is the segment fraction of component $i$ defined in terms of the hard-core volumes of the pure components, and $S_{1} / S_{2}$ is the ratio of the surface area of a solvent molecule and that of a polymer segment having the same hard-core volume as the solvent molecule. Accordingly, the two distinct volume fractions cited above are correlated by

$$
\phi_{2}=\frac{V_{2} / \tilde{v}_{2}}{V_{2} / \tilde{v}_{2}+V_{1} / \tilde{v}_{1}}
$$

where $V_{1}=1-V_{2}$

In addition, $\tilde{v}$ is also concentration dependent as

$$
\tilde{v}^{1 / 3}=1+\tilde{T} \tilde{v}^{4 / 3}
$$

are the atmospheric pressure.

Equations 1 and 2 lead to a useful expression for $\chi$. This equation is conventionally applied to gain information on $X_{12}$ and $Q_{12}$ from the $\chi$ data obtained over a range of $\phi_{2}$. However, in the limit of infinite dilution, we have

$$
\begin{aligned}
\chi_{0}= & \lim _{v_{2} \rightarrow 0} \chi \\
= & \frac{M_{1} \bar{v}_{1}}{R T}\left\{\left(\frac{3 A^{2}}{2 p_{1}^{*}}\right)\left[\left(\tilde{v}_{1}^{1 / 3}-1\right)^{-1}-3\right]\right. \\
& \left.+\left(\frac{S_{2}}{S_{1} \tilde{v}_{2}}\right)^{2}\left(X_{12}-T \tilde{v}_{1} Q_{12}\right)\right\}
\end{aligned}
$$

where

$$
A=\frac{\tilde{v}_{1}\left[P_{2}^{*}\left(\tilde{T}_{2}-\tilde{T}_{1}\right)+\tilde{T}_{1} X_{12} S_{2} / S_{1}\right]}{\tilde{v}_{2}\left(1-4 \tilde{T}_{1} \tilde{v}_{1}\right)}
$$

Alternatively, eq 7 may be derived by the following approach. According to the equationof-state theory the parameter $\chi$ can be redesignated and redefined by

$$
\chi^{\prime}=\mu_{1}^{\mathrm{R}} / R T \phi_{2}^{2}
$$

Comparing eq 8 and 1 results in

$$
\chi=\left(\frac{V_{2}}{\phi_{2}}\right)^{-2} \chi^{\prime}
$$

which leads to

$$
\chi_{0}=\left(\frac{\tilde{v}_{1}}{\tilde{v}_{2}}\right)^{2} \chi_{1}
$$

with the aid of eq 5 , where

$$
\chi_{1}=\lim _{\phi_{2} \rightarrow 0} \chi^{\prime}
$$

The expression for $\chi_{1}$ has been developed earlier, ${ }^{7}$ and it may be introduced into eq 10 to obtain eq 7 by merely making some appropriate substitutions. Hence, $\chi_{0}$ is within the framework of the Flory's equation-of-state thermodynamics. In any case, eq 7 facilitates the interpretation of $\chi_{0}$ data readily obtainable by various experimental techniques.

\section{RESULTS AND DISCUSSION}

The present analysis follows the standard procedure to secure the equation-of-state parameters. ${ }^{5}$ In this connection, it is crucial to know the drift of the specific volume, thermal expansion coefficient and thermal pressure coefficient of each component, and the ratio $S_{1} / S_{2}$ with temperature. As such, we have collected from the reliable sources the required information for polystyrene (PS), ${ }^{8}$ polyisobutylene (PIB), ${ }^{9}$ and various solvents including toluene, ${ }^{10}$ cyclohexane, ${ }^{11,12}$ and benzene. ${ }^{12,13}$ The polymer-solvent interactions of these components are then studies with the 

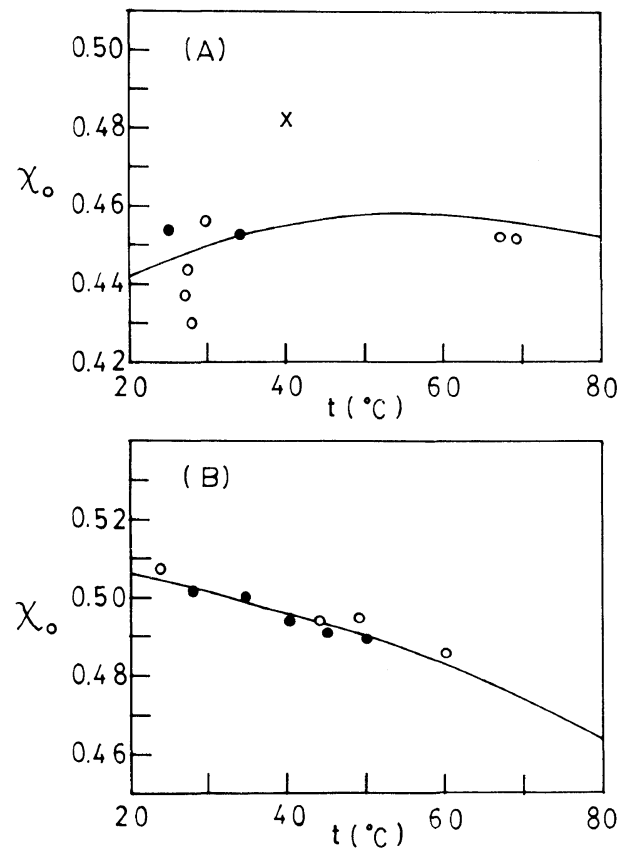

Figure 1. (A) Plot of $\chi_{0}$ against $t$ for polystyrene in toluene. (B) Plot of $\chi_{0}$ against $t$ for polystyrene in cyclohexane. In these figures, the data points are obtained by means of light scattering method $(x)$, osmometry $(O)$, and visometry $(\bigcirc)$, whereas the curves are predicted by the equation-of-state theory.

results presented below.

Figure 1 demonstrates the plots of $\chi_{0}$ against centigrade temperature, $t$, for PS in good and poor solvents. The values of $\chi_{0}$ are derived from the measurements of light scattering, osmotic pressure, and dilute-solution viscosity. Details of these determinations have been outlined, ${ }^{14}$ except the last method which is based on an expression established recently for $\chi_{0}$ in terms of the Mark-Houwink-Sakurada exponent, ${ }^{16}$ and other molecular characteristics. The data points are fitted to eq 7 by means of a non-linear least-squares algorithm to obtain $X_{12}=9.90 \mathrm{~J} \mathrm{ml}^{-1}, Q_{12}=5.2 \times 10^{-3} \mathrm{~J} \mathrm{~m}$ $1^{-1} \mathrm{~K}^{-1}$ for PS in toluene, and $X_{12}=68.7 \mathrm{~J}$ $\mathrm{ml}^{-1}, Q_{12}=1.38 \times 10^{-1} \mathrm{~J} \mathrm{ml}^{-1} \mathrm{~K}^{-1}$ for PS in cyclohexane. Throughout these calculations, the values of $S_{1} / S_{2}$ are equal to 1.087 and 2.000 at $25^{\circ} \mathrm{C}$ as reported for the first and second systems, respectively. ${ }^{10,17}$ On the basis of these

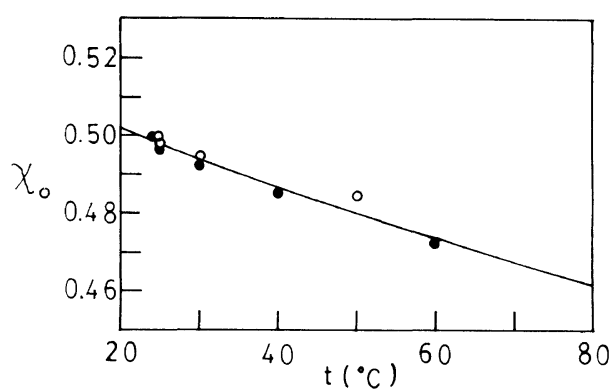

Figure 2. Plot of $\chi_{0}$ against $t$ for polyisobutylene in benzene. Symbols and curve are as in Figure 1.

results, eq 7 predicts the curves shown in Figure 1. Clearly, the theoretical and experimental values of $\chi_{0}$ are reasonably close, except for a couple of erratic measurements for PS in toluene at 28 and $40^{\circ} \mathrm{C}$. The same situation is witnessed for PIB in benzene as displayed in Figure 2. This particular pair yields $X_{12}=44.9$ $\mathrm{J} \mathrm{mol}^{-1}, Q_{12}=3.7 \times 10^{-2} \mathrm{~J} \mathrm{~mol}^{-1} \mathrm{~K}^{-1}$ based on $S_{1} / S_{2}=1.724$ at $25^{\circ} \mathrm{C}^{18}$

Flory and coworkers also have employed the equation-of-state theory to study the thermodynamic interactions in PS-cyclohexane and PIB-benzene solutions, but, by varying the polymer concentration instead. The former system results in $X_{12}=42.0 \mathrm{~J} \mathrm{~mol}^{-1}, Q_{12}=$ $2.3 \times 10^{-2} \mathrm{~J} \mathrm{ml}^{-1} \mathrm{~K}^{-1},{ }^{17}$ whereas the latter pair renders $X_{12}=41.8 \mathrm{~J} \mathrm{ml}^{-1}, Q_{12}=0 .{ }^{18} \mathrm{It}$ follows that eq 7 overestimates the $\chi_{0}$ considerably over the $T$ range from 20 to $80^{\circ} \mathrm{C}$. The foregoing free volume model has been modified by marking allowance for the departure from the linear relationship between the number of external degrees of freedom per segment in the mixture and the concentration. Accordingly, $\mu_{1}^{\mathrm{R}}$ is characterized by $X_{12}$ and a new parameter $C_{12}$, which accounts for the foregoing deviation. Shiomi et al. ${ }^{10}$ have investigated the thermodynamic behavior of PS-toluene solutions covering $\phi_{2} \leq 20 \%$ by osmometry, and concluded $X_{12}=1.2 \mathrm{~J} \mathrm{ml}^{-1}$, $C_{12}=0.0102$. The least-squares fitting of their $\chi^{\prime}\left(\phi_{2}\right)$ data to the Flory's equation-of-state theory ${ }^{7}$ suggests $X_{12}=10.0 \mathrm{~J} \mathrm{ml}^{-1}$ and $Q_{12}=0$. Again, these values are incompatible with the 


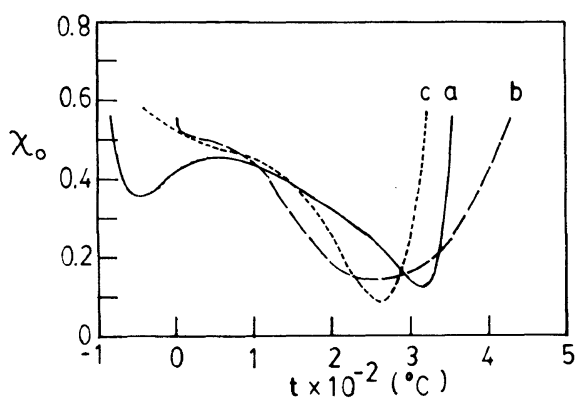

Figure 3. Temperature dependences of $\chi_{0}$ as predicted by the Flory's equation-of-state theory for (a) PS-toluene, (b) PS-cyclohexane, and (c) PIB-benzene.

results obtained by the present analysis for this particular polymer solvent pair, in that they lead to significantly higher $\chi_{0}$.

In plotting the $\chi^{\prime} / V_{1}^{*}$ against $t$ for a hypothetical polymer-solvent pair with $X_{12}>$ 0 , Patterson and Robard ${ }^{6}$ have demonstrated a characteristic U-shaped curve intersecting with the line corresponding to $\chi^{\prime}=\chi_{\mathrm{c}}^{\prime}$ at the LCST and UCST, where $V_{1}^{*}$ and $\chi_{\mathrm{c}}^{\prime}$ are respectively the core volume of solvent and the critical $\chi^{\prime}$. However, Figures $1(\mathrm{~A})$ and (B) display two convex curves instead, indicating that no LCST and UCST occurring at $\chi_{0}=1 / 2$ are possible as inferred from the foregoing observation. This "peculiar" behavior of the PS solutions may be due to the fact that the present analysis duly considers the temperature effects on the various characteristic parameters particularly $\tilde{v}_{i}^{*}, T_{i}^{*}$, and $P_{i}^{*}$, which unfortunately have been ignored altogether in the previous case. In fact, the changes of $\chi_{0}$ with $t$ do not necessarily follow the proposed U-shaped function cited above as shown in Figure 3, which extends the curves presented in Figures 1 and 2 to cover the LCST and UCST at $\chi_{0}=1 / 2$ for the systems of interest. Incidentally, the literature values of the LCST for the systems PS-toluene, PS-cyclohexane, and PIB-benzene are respectively $277^{\circ} \mathrm{C},{ }^{19}$ $213^{\circ} \mathrm{C},{ }^{19}$ and $260^{\circ} \mathrm{C} .{ }^{20}$ Clearly, the theoretical predictions of these physical parameters obtained from Figure 3, are by no means satisfactory. However, the observed and predicted theta temperature, $T_{\theta}$, based on the UCST for the last two systems are indeed reasonably consistent (see Figures 1(B) and 2).

Basically, the two exchange parameters of the Flory theory serve as the adjustable constants to optimize the agreement with experiment. Hence, it is not unexpected to have inconsistent values of $X_{12}$ and $Q_{12}$ resulting from distinct sets of thermodynamic data for a particular polymeric system, as mentioned in the present study. In addition, the present analysis shows that the predicted $T_{\theta}$ 's occurring at extreme temperatures are rather unreliable. This implies that the constancy of exchange parameters cited may be applied only over a limited range of temperatures. Despite these inadequacies, the equation-of-state theory reproduces the observed temperature dependence of $\chi_{0}$ satisfactorily. Indeed, this achievement is particularly significant for polymers in good solvents, which may defy the classical description of $\chi_{0}$ represented by a linear function of the reciprocal of temperature. ${ }^{21,22}$

Acknowledgement. The author thanks one of the reviewers for his helpful comments.

\section{REFERENCES}

1. P. J. Flory, "Principles of Polymer Chemistry," Cornell University Press, Ithaca, New York, N.Y., 1953, Chapter 12.

2. G. Ten Brinke, F. E. Karasz, and W. J. MacKnight, Macromolecules, 16, 1827 (1983).

3. P. J. Flory, Discuss Faraday Soc., 49, 7 (1970).

4. L. P. McMaster, Macromolecules, 6, 760 (1973).

5. G. Ten Brinke, A. Eshuis, E. Roerdink, and G. Challa, Macromolecules, 14, 867 (1981).

6. D. Patterson and A. Robard, Macromolecules, 11, 690 (1978).

7. P. J. Flory and H. Hocker, Trans. Faraday Soc., 67, 2258 (1971).

8. H. Hocker, G. J. Blake, and P. J. Flory, Trans. Faraday Soc., 67, 2251 (1971).

9. B. E. Eichinger and P. J. Flory, Macromolecules, 1, 285 (1968).

10. T. Shiomi, K. Kohno, K. Yonada, T. Tomita, M. Miya, and K. Imai, Macromolecules, 18, 414 (1985).

11. S. E. Wood and J. A. Gray, J. Am. Chem. Soc., 74, 
3729 (1952).

12. G. A. Holder and W. Whalley, Trans. Faraday Soc., 58, 2095 (1962).

13. S. E. Wood and J. B. Brusie, J. Am. Chem. Soc., 65, 1891 (1943).

14. R. A. Orwoll, Rubber Chem. Technol., 50, 451 (1977).

15. K. K. Chee, Polymer, 28, 977 (1987).

16. M. Kurata, Y. Tsunashima, M. Iwama, and K. Kamada, in "Polymer Handbook," 2nd ed, J. Bandrup and E. H. Immergut, Ed., Wiley- Interscience, New York, N. Y., 1975, Chapter 4.

17. H. Hocker, H. Shih, and P. J. Flory, Trans. Faraday
Soc., 67, 2275 (1971).

18. B. E. Eichinger and P. J. Flory, Trans. Faraday Soc., 64, 2053 (1968).

19. S. Saeki, N. Kuwahara, S. Konno, and M. Kaneko, Macromolecules, 6, 246 (1973).

20. A. H. Liddell and F. L. Swinton, Discuss Faraday Soc., 49, 115 (1970).

21. M. L. Huggins and H. Okamoto, in "Polymer Fraction," M. J. R. Cantow, Ed, Academic Press, New York, N.Y., 1967, Chapter A.

22. T. P. Russell, R. P. Hjelm, Jr., and P. A. Seager, Macromolecules, 23, 890 (1990). 